\title{
Symmetric Small-Molecules with Acceptor-Donor-Acceptor Architecture for Efficient Visible-Light Driven Hydrogen Production: Optical and Thermodynamic Aspects
}

Giane B. Damas, ${ }^{a}$ Fredrik von Kieseritzky, ${ }^{b}$ Jonas Hellberg ${ }^{\mathrm{b}}$, Cleber F. N. Marchiori, ${ }^{* c}$ C. Moyses Araujo*a

a Materials Theory Division, Department of Physics and Astronomy, Angström Laboratory, Uppsala University, Box 516, 75120 Uppsala, Sweden.

${ }^{b}$ Arubedo AB, Södra Hamnvägen 9, 11451 Stockholm, Sweden

${ }^{c}$ Department of Chemistry - Angström Laboratory, Uppsala University, Box 538, 75121

Uppsala, Sweden.

Table S1 - Comparison between the theoretical and experimental (in parenthesis) values for the potential of $\left(\mathrm{SM}^{+} / \mathrm{SM}\right)$ and $\left(\mathrm{SM} / \mathrm{SM}^{-}\right)$redox pairs and absorption maximum. Level of theory: M06/6-311G(d,p). Solvent: dichloromethane.

\begin{tabular}{cccc}
\hline Molecule & $\phi_{S M^{+} / S M}(\mathrm{~V})$ & $\phi_{S M / S M^{-}}(\mathrm{V})$ & Abs. Max. $(\mathrm{nm})$ \\
\hline DCAO3T(BDT)3T $^{1}$ & $5.07(5.11)$ & $3.09(3.54)$ & $406(478)$ \\
DCAO3TSi $^{2}$ & $4.88(4.95)$ & $3.08(3.26)$ & $580(518)$ \\
DTSi(FBTAT $\left._{2}\right)_{2}{ }^{3}$ & $4.84(5.42)$ & $2.96(3.47)$ & $588(516)$ \\
FDICTF $^{4}$ & $5.42(5.43)$ & $3.59(3.71)$ & $581(665)$ \\
FDNCTF $^{4}$ & $5.44(5.42)$ & $3.63(3.73)$ & $599(697)$ \\
\hline
\end{tabular}

Table S2 - Comparison between the theoretical and experimental (in parenthesis) values for the potential of $\left(\mathrm{SM}^{+} / \mathrm{SM}\right)$ and $\left(\mathrm{SM} / \mathrm{SM}^{-}\right)$redox pairs and absorption maximum. Level of theory: M06/6-311G(d,p). Solvent: water.

\begin{tabular}{cccc}
\hline Molecule & $\phi_{S M^{+} / S M}(\mathrm{~V})$ & $\phi_{S M / S M^{-}}(\mathrm{V})$ & Abs. Max. $(\mathrm{nm})$ \\
\hline DCAO3T(BDT)3T $^{1}$ & $5.07(5.11)$ & $3.35(3.54)$ & $406(478)$ \\
DCAO3TSi $^{2}$ & $4.84(4.95)$ & $3.33(3.26)$ & $580(518)$ \\
DTSi(FBTAT $\left._{2}\right)_{2}{ }^{3}$ & $4.86(5.42)$ & $3.17(3.47)$ & $588(516)$ \\
FDICTF $^{4}$ & $5.51(5.43)$ & $3.82(3.71)$ & $581(665)$ \\
FDNCTF $^{4}$ & $5.53(5.42)$ & $3.87(3.73)$ & $599(697)$ \\
\hline
\end{tabular}


Table S3-Electronic energies (in hartrees) in aqueous environment obtained after single-point calculations with PCM or SMD implicit solvent model.

\begin{tabular}{lcccccccc}
\hline Systems & \multicolumn{2}{c}{ Neutral } & \multicolumn{2}{c}{ Anion } & \multicolumn{2}{c}{ Cation } & \multicolumn{2}{c}{ Excited State } \\
& PCM & SMD & PCM & SMD & PCM & SMD & PCM & SMD \\
\hline SM1-H & -4981.52737 & -4981.52592 & -4981.64161 & -4981.63912 & -4981.35076 & -4981.35256 & -4981.52277 & -4981.52124 \\
SM1-DFM & -6233.44620 & -6233.44077 & -6233.56540 & -6233.55780 & -6233.26754 & -6233.26595 & -6233.44135 & -6233.43579 \\
SM2-H & -5045.68991 & -5045.69542 & -5045.81248 & -5045.81678 & -5045.50859 & -5045.51761 & -5045.68604 & -5045.69148 \\
SM2-DFM & -6297.59650 & -6297.59740 & -6297.72387 & -6297.72273 & -6297.41104 & -6297.41597 & -6297.59231 & -6297.59345 \\
SM3-H & -5437.08625 & -5437.08940 & -5437.19405 & -5437.19652 & -5436.91611 & -5436.92225 & -5437.07892 & -5437.08177 \\
SM3-DFM & -6689.00910 & -6689.00762 & -6689.12338 & -6689.11996 & -6688.83360 & -6688.83621 & -6688.99732 & -6688.99780 \\
SM4-H & -5501.24876 & -5501.25880 & -5501.36548 & -5501.37468 & -5501.07566 & -5501.08910 & -5501.23401 & -5501.24421 \\
SM4-DFM & -6753.15931 & -6753.16294 & -6753.28557 & -6753.28854 & -6752.98294 & -6752.99122 & -6753.14659 & -6753.15249 \\
\hline
\end{tabular}


Table S4- Potentials for the $\left(\mathrm{SM}^{+} / \mathrm{SM}\right)$ and $\left(\mathrm{SM} / \mathrm{SM}^{-}\right)$redox pairs obtained in aqueous environment via Gibbs free energies of reaction $\left(\Delta \mathrm{G}_{\text {(solv) }}\right)$ environment or using the electronic energies $(\triangle \mathrm{SCF})$. Level of theory: M06/6-311G(d,p).

\begin{tabular}{lcccc}
\hline Systems & \multicolumn{2}{c}{$\mathbf{S M}^{+} / \mathbf{S M}(\mathbf{V}$ vs. SHE $)$} & \multicolumn{2}{c}{$\mathbf{S M} / \mathbf{S M}(\mathbf{V}$ vs. SHE) } \\
& $\Delta \mathbf{G}_{(\mathbf{s o l v})}$ & $\Delta \mathbf{S C F}$ & $\Delta \mathbf{G}_{(\mathbf{s o l v})}$ & $\Delta \mathbf{S C F}$ \\
\hline SM1-H & +0.42 & +0.37 & -1.32 & -1.33 \\
SM1-DFM & +0.47 & +0.42 & -1.16 & -1.20 \\
SM2-H & +0.49 & +0.49 & -1.06 & -1.11 \\
SM2-DFM & +0.66 & +0.61 & -0.90 & -0.97 \\
SM3-H & +0.22 & +0.19 & -1.45 & -1.51 \\
SM3-DFM & +0.33 & +0.34 & -1.19 & -1.33 \\
SM4-H & +0.26 & +0.27 & -1.18 & -1.26 \\
SM4-DFM & +0.43 & +0.36 & -0.92 & -1.00 \\
\hline
\end{tabular}

Table S5- Potentials for the $\left(\mathrm{SM}^{*} / \mathrm{SM}^{-}\right)$and $\left(\mathrm{SM}^{+} / \mathrm{SM}^{*}\right)$ redox pairs obtained in aqueous environment via Gibbs free energies of reaction $\left(\Delta \mathrm{G}_{(\mathrm{solv})}\right)$ environment or using the electronic energies $(\triangle \mathrm{SCF})$. Level of theory: M06/6-311G(d,p).

\begin{tabular}{lcccc}
\hline Systems & \multicolumn{2}{c}{$\mathbf{S M}^{*} / \mathbf{S M}^{-}(\mathbf{V}$ vs. SHE $)$} & \multicolumn{2}{c}{$\mathbf{S M}^{+} / \mathbf{S M}^{*}(\mathbf{V}$ vs. SHE) } \\
& $\Delta \mathbf{G}$ & $\Delta \mathbf{S C F}$ & $\Delta \mathbf{G}$ & $\Delta \mathbf{S C F}$ \\
\hline SM1-H & +0.43 & +0.39 & -1.32 & -1.36 \\
SM1-DFM & +0.48 & +0.44 & -1.21 & -1.17 \\
SM2-H & +0.65 & +0.62 & -1.21 & -1.23 \\
SM2-DFM & +0.80 & +0.72 & -1.04 & -1.09 \\
SM3-H & +0.22 & +0.16 & -1.45 & -1.48 \\
SM3-DFM & +0.18 & +0.05 & -1.04 & -1.04 \\
SM4-H & +0.31 & +0.24 & -1.22 & -1.24 \\
SM4-DFM & +0.31 & +0.15 & -0.80 & -0.79 \\
\hline
\end{tabular}




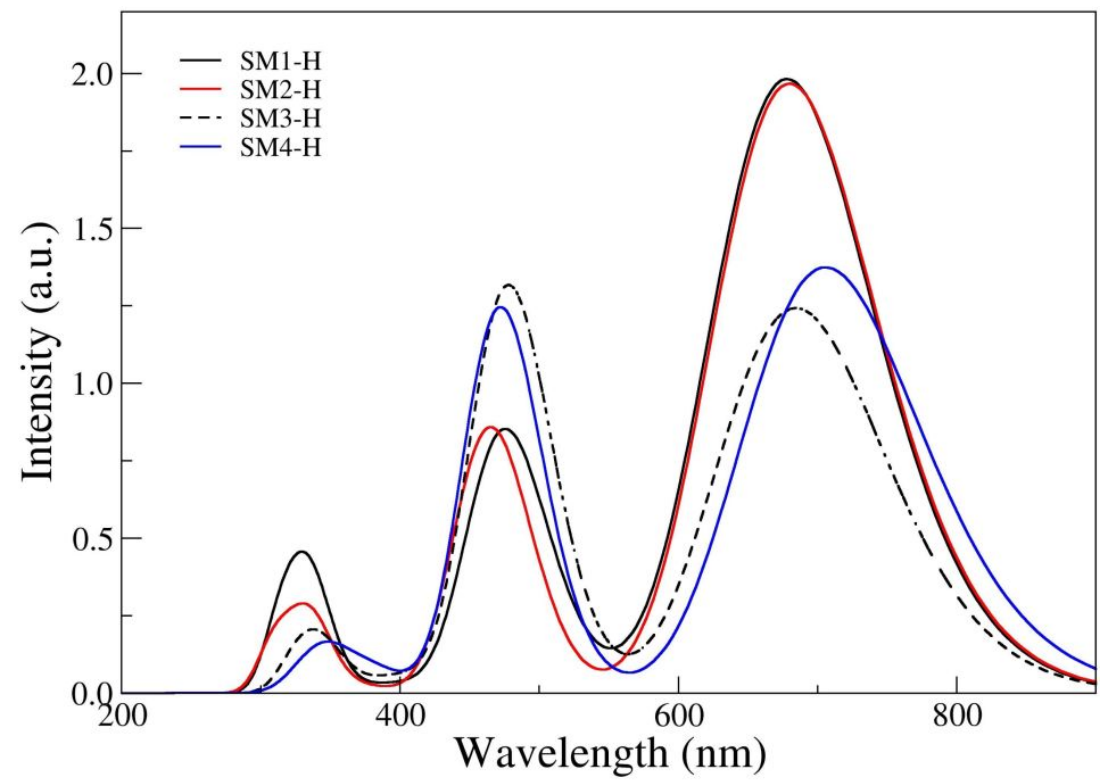

Figure S1- Theoretical UV-Vis spectra for SM-H compounds with intensities weighed by the oscillator strength of each electronic transition.

Table S6- Description of the orbital contribution for the main single electronic transitions and obtained at the range 200-1000 $\mathrm{nm}$ within TDDFT methods. The optical gap for these compounds is displayed in the last column. Level of theory: M06/6-311G(d,p).

\begin{tabular}{|c|c|c|c|c|}
\hline & $\begin{array}{l}\text { Wavelength } \\
(\mathrm{nm})\end{array}$ & f & Contributions & Optical gap (eV) \\
\hline SM1-H & $\begin{array}{l}\mathrm{S}_{1}=677.99 \\
\mathrm{~S}_{4}=477.52 \\
\mathrm{~S}_{5}=470.26 \\
\mathrm{~S}_{12}=337.17\end{array}$ & $\begin{array}{l}1.977 \\
0.453 \\
0.376 \\
0.293\end{array}$ & $\begin{array}{l}\mathrm{H} \rightarrow \mathrm{L}(93 \%) \\
\mathrm{H}-1 \rightarrow \mathrm{L}+1(53 \%) \\
\mathrm{H} \rightarrow \mathrm{L}+2(65 \%) \\
\mathrm{H} \rightarrow \mathrm{L}+4(72 \%)\end{array}$ & 1.829 \\
\hline SM2-H & $\begin{array}{l}\mathrm{S}_{1}=679.59 \\
\mathrm{~S}_{4}=464.74 \\
\mathrm{~S}_{12}=337.60\end{array}$ & $\begin{array}{l}1.961 \\
0.821 \\
0.202\end{array}$ & $\begin{array}{l}\mathrm{H} \rightarrow \mathrm{L}(95 \%) \\
\mathrm{H} \rightarrow \mathrm{L}+2(74 \%) \\
\mathrm{H} \rightarrow \mathrm{L}+4(72 \%)\end{array}$ & 1.824 \\
\hline SM3-H & $\begin{array}{l}\mathrm{S}_{1}=684.47 \\
\mathrm{~S}_{4}=479.30 \\
\mathrm{~S}_{14}=344.76\end{array}$ & $\begin{array}{l}1.307 \\
1.209 \\
0.127\end{array}$ & $\begin{array}{l}\mathrm{H} \rightarrow \mathrm{L}(94 \%) \\
\mathrm{H} \rightarrow \mathrm{L}+2(61 \%) \\
\mathrm{H} \rightarrow \mathrm{L}+4(91 \%)\end{array}$ & 1.811 \\
\hline SM4-H & $\begin{array}{l}\mathrm{S}_{1}=706.42 \\
\mathrm{~S}_{4}=472.61 \\
\mathrm{~S}_{12}=350.06\end{array}$ & $\begin{array}{l}1.369 \\
1.157 \\
0.100\end{array}$ & $\begin{array}{l}\mathrm{H} \rightarrow \mathrm{L}(95 \%) \\
\mathrm{H} \rightarrow \mathrm{L}+2(74 \%) \\
\mathrm{H} \rightarrow \mathrm{L}+4(82 \%)\end{array}$ & 1.755 \\
\hline SM1-DFM & $\begin{array}{l}\mathrm{S}_{1}=718.96 \\
\mathrm{~S}_{4}=486.74 \\
\mathrm{~S}_{5}=467.34 \\
\mathrm{~S}_{18}=318.34\end{array}$ & $\begin{array}{l}1.773 \\
0.621 \\
0.505 \\
0.154\end{array}$ & $\begin{array}{l}\mathrm{H} \rightarrow \mathrm{L}(93 \%) \\
\mathrm{H}-1 \rightarrow \mathrm{L}+1(58 \%) \\
\mathrm{H} \rightarrow \mathrm{L}+2(69 \%) \\
\mathrm{H}-2 \rightarrow \mathrm{L}+2(67 \%)\end{array}$ & 1.725 \\
\hline SM2-DFM & $\begin{array}{l}S_{1}=687.96 \\
S_{3}=468.68\end{array}$ & $\begin{array}{l}1.881 \\
0.274\end{array}$ & $\begin{array}{l}\mathrm{H} \rightarrow \mathrm{L}(95 \%) \\
\mathrm{H}-1 \rightarrow \mathrm{L}(71 \%)\end{array}$ & 1.802 \\
\hline
\end{tabular}




\begin{tabular}{lllll} 
& $\mathrm{S}_{4}=463.54$ & 0.677 & $\mathrm{H} \rightarrow \mathrm{L}+2(64 \%)$ & \\
& $\mathrm{S}_{13}=338.15$ & 0.122 & $\mathrm{H} \rightarrow \mathrm{L}+4(72 \%)$ & \\
\hline SM3-DFM & $\mathrm{S}_{1}=704.26$ & 1.250 & $\mathrm{H} \rightarrow \mathrm{L}(93 \%)$ & \\
& $\mathrm{S}_{3}=488.40$ & 0.634 & $\mathrm{H}-1 \rightarrow \mathrm{L}+1(36 \%)$ & \\
& & & $\mathrm{H}-1 \rightarrow \mathrm{L}(28 \%)$ & \\
& & & & \\
& $\mathrm{S}_{4}=484.50$ & 0.707 & $\mathrm{H}-1 \rightarrow \mathrm{L}(44 \%)$ & 1.722 \\
\hline SM4-DFM & $\mathrm{S}_{1}=717.88$ & 1.102 & $\mathrm{H} \rightarrow \mathrm{L}(94 \%)$ & \\
& $\mathrm{S}_{3}=472.77$ & 1.214 & $\mathrm{H} \rightarrow \mathrm{L}+2(84 \%)$ & \\
\hline
\end{tabular}
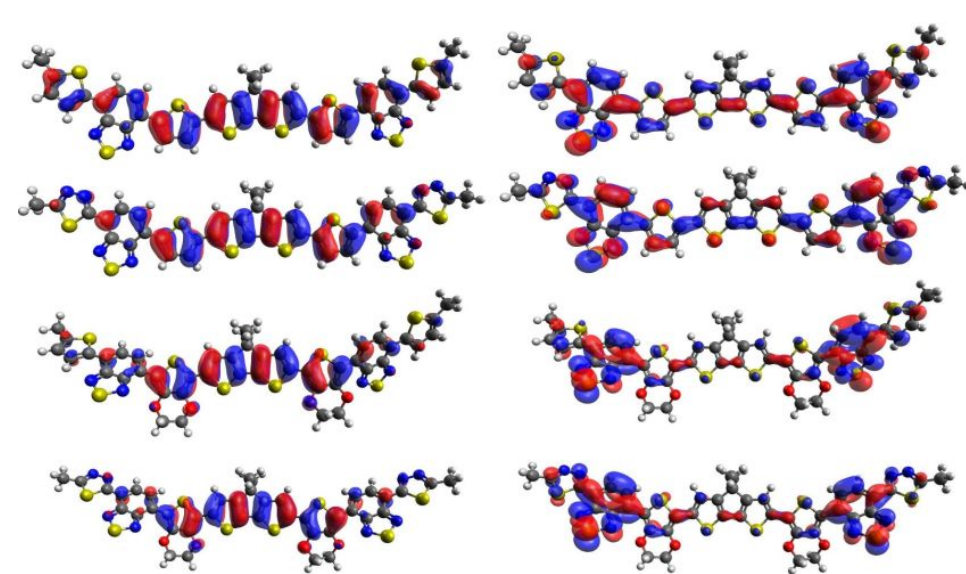

НОМО

LUMO
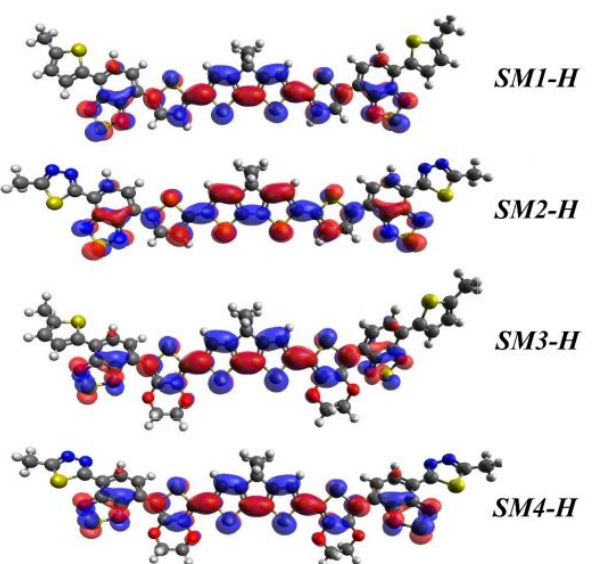

$\mathrm{LUMO}+2$

Figure S2- Spatial distribution of the molecular orbitals that are involved in the main electronic transitions. Isovalue:0.02.

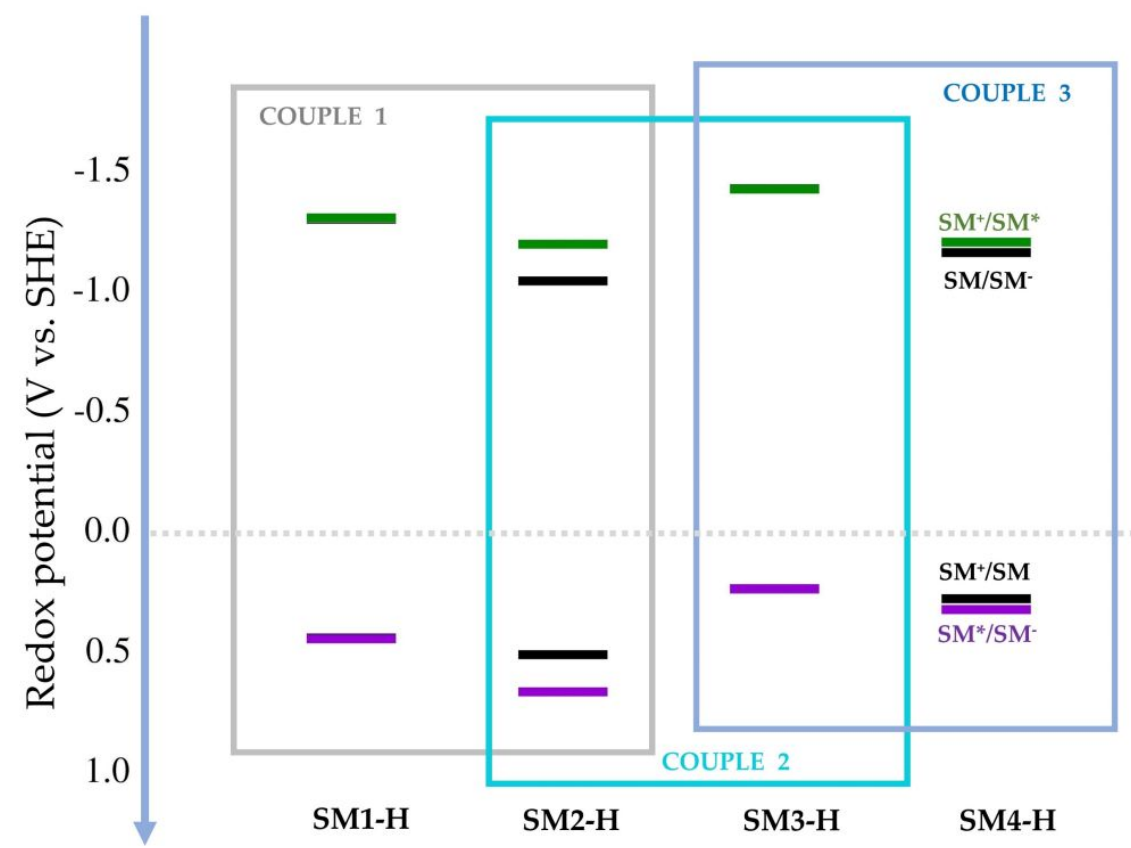

Figure S3 - Redox potentials indicated by the black and colorful horizontal bars for SM-compounds, with highlight for the possibility of forming photocatalytic couples. All values are referred to SHE (set at 0 ). 
Table S7- Cooperative photocatalytic systems with the driving force (in $\mathrm{eV}$ ) for the charge transfer process in cascade. Level of theory: M06/6-311G(d,p).

\begin{tabular}{|c|c|c|c|}
\hline Couple & Photoexcitation & $\begin{array}{l}\text { Electron transfer } \\
\mathbf{A} \rightarrow \mathbf{B} \\
\Delta \mathbf{G}(\mathrm{eV})\end{array}$ & $\begin{array}{l}\text { Hole transfer } \\
\mathrm{B} \rightarrow \mathbf{A} \\
\Delta \mathbf{G}(\mathrm{eV})\end{array}$ \\
\hline \multirow[t]{2}{*}{1} & $\begin{array}{l}\text { (A)SM1-DFM } \\
\lambda_{1}=718.96 \mathrm{~nm}\end{array}$ & 0.26 & - \\
\hline & $\begin{array}{l}\text { (B) SM2-DFM } \\
\lambda_{1}=687.96 \mathrm{~nm}\end{array}$ & - & 0.19 \\
\hline \multirow[t]{2}{*}{2} & $\begin{array}{l}\text { (A)SM3- DFM } \\
\lambda_{1}=704.26 \mathrm{~nm}\end{array}$ & 0.29 & - \\
\hline & $\begin{array}{l}\text { (B)SM2-DFM } \\
687.96 \mathrm{~nm}\end{array}$ & - & 0.33 \\
\hline \multirow[t]{2}{*}{3} & $\begin{array}{l}\text { (A)SM3-DFM } \\
\lambda_{1}=704.26 \mathrm{~nm}\end{array}$ & 0.27 & - \\
\hline & $\begin{array}{l}\text { (B) SM4-DFM } \\
\lambda_{1}=717.88 \mathrm{~nm}\end{array}$ & - & 0.10 \\
\hline
\end{tabular}

Table S8- Geometric parameters at different catalytic sites upon hydrogenation in SM-DFM compounds. $\theta$ corresponds to the dihedral angle between the hydrogenated unit (BT or Tz) and the nearest unit (EDOT, thiophene or BT), with labels $i$ and $f$ indicating initial and final molecule (after hydrogenation), respectively. The last column provides the hydrogen binding free energies for these groups. Level of theory: M06/6-311G(d,p).

\begin{tabular}{|c|c|c|c|c|c|c|}
\hline System/Site & N-H $(\AA)$ & $\mathrm{O} \cdots \mathrm{H}(\AA)$ & $\theta_{\mathrm{i}}\left(^{\circ}\right)$ & $\theta_{\mathrm{f}}\left(^{\circ}\right)$ & $|\Delta \theta|\left(^{\circ}\right)$ & $\Delta \mathrm{G}_{\mathrm{H}}(\mathrm{eV})$ \\
\hline $\begin{array}{l}\text { SM1-DFM } \\
\text { (BT site) }\end{array}$ & 1.019 & - & -8.3 & -27.7 & 19.4 & 0.64 \\
\hline $\begin{array}{l}\text { SM2-DFM } \\
\text { (BT-site) }\end{array}$ & 1.019 & - & 16.1 & 34.4 & 17.3 & 0.58 \\
\hline (Tz-site) & 1.014 & $2.038(\mathrm{DFM})$ & -28.4 & -0.7 & 27.7 & 0.14 \\
\hline $\begin{array}{l}\text { SM3-DFM } \\
\text { (BT-site) }\end{array}$ & 1.022 & $1.903(\mathrm{EDOT})$ & 41.4 & 38.7 & 2.7 & 0.48 \\
\hline $\begin{array}{l}\text { SM4-DFM } \\
\text { (BT site) }\end{array}$ & 1.022 & 1.901(EDOT) & 41.2 & 37.8 & 3.4 & 0.40 \\
\hline Tz site & 1.014 & 2.053 (DFM) & -25.1 & 0.8 & 25.9 & 0.08 \\
\hline
\end{tabular}

\section{References}

(1) Liu, Y.; Wan, X.; Wang, F.; Zhou, J.; Long, G.; Tian, J.; Chen, Y. High-Performance Solar Cells Using a Solution-Processed Small Molecule Containing Benzodithiophene Unit. Adv. Mater. 2011, 23, 5387-5391.

(2) Zhou, J.; Wan, X.; Liu, Y.; Long, G.; Wang, F.; Li, Z.; Zuo, Y.; Li, C.; Chen, Y. A Planar Small Molecule with Dithienosilole Core for High Efficiency Solution-Processed Organic Photovoltaic Cells. Chem. Mater. 2011, 23, 4666-4668.

(3) Keshtov, M. L.; Godovsky, D. Y.; Kuklin, S. A.; Lee, J.; Kim, J.; Lim, B.; Lee, H. K.; 
Biswas, S.; Koukaras, E. N.; Sharma, G. D. Design, Synthesis and Photophysical Properties of D1-A-D2-A-D1-Type Small Molecules Based on Fluorobenzotriazole Acceptor and Dithienosilole Core Donor for Solution Processed Organic Solar Cells. Dye. Pigment. 2016, 132, 387-397.

(4) Feng, H.; Qiu, N.; Wang, X.; Wang, Y.; Kan, B.; Wan, X.; Zhang, M.; Xia, A.; Li, C.; Liu, F.; et al. An A-D-A Type Small-Molecule Electron Acceptor with End-Extended Conjugation for High Performance Organic Solar Cells. Chem. Mater. 2017, 29, 79087917. 